\title{
Convergence of TTS Iterative Method for Non-Hermitian Positive Definite Linear Systems
}

\author{
Cheng-Yi Zhang, ${ }^{1}$ Yu-Qian Yang, ${ }^{2}$ and Qiang Sun $^{3}$ \\ ${ }^{1}$ School of Science, Xian Polytechnic University, Xian, Shaanxi 710048, China \\ ${ }^{2}$ School of Mathematics and Statistics, Xidian University, Xian, Shaanxi 710075, China \\ ${ }^{3}$ Department of Electronic Engineering, Xi'an University of Technology, Xian, Shaanxi 710048, China
}

Correspondence should be addressed to Cheng-Yi Zhang; cyzhang08@126.com

Received 26 February 2014; Accepted 28 April 2014; Published 19 May 2014

Academic Editor: Suh-Yuh Yang

Copyright (C) 2014 Cheng-Yi Zhang et al. This is an open access article distributed under the Creative Commons Attribution License, which permits unrestricted use, distribution, and reproduction in any medium, provided the original work is properly cited.

The TTS iterative method is proposed to solve non-Hermitian positive definite linear systems and some convergence conditions are presented. Subsequently, these convergence conditions are applied to the ALUS method proposed by Xiang et al. in 2012 and comparison of some convergence theorems is made. Furthermore, an example is given to demonstrate the results obtained in this paper.

\section{Introduction}

Many problems in scientific computing give rise to a system of $n$ linear equations in $n$ unknowns,

$$
A x=b, \quad A=\left(a_{i j}\right) \in \mathbb{C}^{n \times n} \text { nonsingular, } \quad b, x \in \mathbb{C}^{n}
$$

where $A$ is a large and sparse non-Hermitian matrix. In this paper we consider the important case where $A$ is positive definite; that is, the Hermitian part $H=\left(A+A^{*}\right) / 2$ is Hermitian positive definite, where $A^{*}$ denotes the conjugate transpose of the matrix $A$. Large and sparse systems of this type arise in many applications, including discretizations of convectiondiffusion problems [1], regularized weighted least-squares problems [2], real-valued formulations of certain complex symmetric systems [3].

There have been several studies on the convergence of splitting iterative methods for non-Hermitian positive definite linear systems. In [4, pages 190-193], some convergence conditions for the splitting of non-Hermitian positive definite matrices have been established. More recently, [5, 6] give some conditions for the convergence of splittings for this class of linear systems.
Recently, there has been considerable interest in the HSS (Hermitian and skew-Hermitian splitting) method introduced by Bai et al. in order to solve non-Hermitian positive definite linear systems; see [7]; we further note the generalizations and extensions of this basic method proposed in [8-13]. Furthermore, these methods and their convergence theories have been shown to apply to (generalized) saddle point problems, either directly or indirectly (as a preconditioner); see $[5,6,8,9,11-16]$.

Continuing in this direction, in this paper we establish new results on splitting methods for solving system (1) iteratively, focusing on a particular class of splittings - the Triangular and Triangular splitting (TTS). According to the idea of HSS method, we construct another alternative iterative method-the TTS method to solve non-Hermitian positive definite linear systems; furthermore, we will prove the convergence of this alternative method.

\section{The TTS Method}

Let $A=\left(a_{i j}\right) \in \mathbb{C}^{n \times n}$ be non-Hermitian positive definite with the Hermitian part $H=\left(A+A^{*}\right) / 2$. We split $A$ as $A=D-$ $L-U$, where $D=\operatorname{diag}\left(a_{11}, a_{22}, \ldots, a_{n n}\right), L$ is strictly lower 
triangular matrix, and $U$ is strictly upper triangular matrix. Let $\mathscr{L}=D / 2-L$ and let $\mathscr{U}=D / 2-U$. Then the splitting

$$
A=\mathscr{L}+\mathscr{U}
$$

is called the Triangular and Triangular splitting (TTS). Subsequently, we give the TTS method.

The TTS Method. Given an initial guess $x^{(0)}$, for $k=0,1,2, \ldots$, until $\left\{x^{(k)}\right\}$ converges, compute

$$
\begin{gathered}
(\alpha I+\mathscr{L}) x^{(k+1 / 2)}=(\alpha I-\mathscr{U}) x^{(k)}+b, \\
(\alpha I+\mathscr{U}) x^{(k+1)}=(\alpha I-\mathscr{L}) x^{(k+1 / 2)}+b,
\end{gathered}
$$

where $\alpha$ is a given positive constant.

Eliminating $x^{(i+1 / 2)}$ from (3), we obtain the iterative process

$$
x^{(k+1)}=T_{\alpha} x^{(k)}+F_{\alpha} b, \quad i=0,1,2, \ldots,
$$

where now $T_{\alpha}=(\alpha I+\mathscr{U})^{-1}(\alpha I-\mathscr{L})(\alpha I+\mathscr{L})^{-1}(\alpha I-\mathscr{U})$ is the iteration matrix of the iterative scheme (4) and $F_{\alpha}=[(\alpha I+$ $\mathscr{L})(\alpha I+\mathscr{U})]^{-1}[(\alpha+1) I+\mathscr{L}]$. It is easy to see that the iterative scheme (3) is convergent if and only if the iterative scheme (4) is convergent. Thus, we only consider the convergence of the iterative scheme (4) and consequently investigate the spectral radius, $\rho\left(T_{\alpha}\right)$, of the iterative matrix $T_{\alpha}$.

\section{Convergence Condition for TTS Method}

In this section, a convergence condition for TTS method is presented to solve non-Hermitian positive definite linear systems. The following lemma will be used in this section.

Lemma 1. Let $A \in \mathbb{C}^{n \times n}$ be non-Hermitian. Then the iteration matrix of the iterative scheme (4) is

$$
T_{\alpha}=\left(\alpha^{2} I+\alpha A+\mathscr{L} \mathcal{U}\right)^{-1}\left(\alpha^{2} I-\alpha A+\mathscr{L} \mathcal{U}\right) .
$$

Proof. Since $A=\mathscr{L}+\mathscr{U}$,

$$
\begin{aligned}
T_{\alpha}= & (\alpha I+\mathscr{U})^{-1}(\alpha I-\mathscr{L})(\alpha I+\mathscr{L})^{-1}(\alpha I-\mathscr{U}) \\
= & 2 \alpha(\alpha I+\mathscr{U})^{-1}(\alpha I+\mathscr{L})^{-1}(\alpha I-\mathscr{U}) \\
& -(\alpha I+\mathscr{U})^{-1}(\alpha I-\mathscr{U}) \\
= & 2 \alpha\left[(\alpha I+\mathscr{U})^{-1}(\alpha I+\mathscr{L})^{-1}(\alpha I-\mathscr{U})-(\alpha I+\mathscr{U})^{-1}\right]+I \\
= & I-2 \alpha(\alpha I+\mathscr{U})^{-1}(\alpha I+\mathscr{L})^{-1}(\mathscr{L}+\mathscr{U}) \\
= & (\alpha I+\mathscr{U})^{-1}(\alpha I+\mathscr{L})^{-1} \\
& \times[(\alpha I+\mathscr{L})(\alpha I+\mathscr{U})-2 \alpha(\mathscr{L}+\mathscr{U})] \\
= & {[(\alpha I+\mathscr{L})(\alpha I+\mathscr{U})]^{-1}\left[\alpha^{2} I-\alpha \mathscr{L}-\alpha \mathscr{U}+\mathscr{L} \mathscr{U}\right) } \\
= & \left(\alpha^{2} I+\alpha A+\mathscr{L} \mathscr{U}\right)^{-1}\left(\alpha^{2} I-\alpha A+\mathscr{L} \mathscr{U}\right),
\end{aligned}
$$

which completes the proof.
Theorem 2. Let $A \in \mathbb{C}^{n \times n}$ be non-Hermitian positive definite with the TTS as in (2). Then the TTS method converges to the unique solution of (1) for any choice of the initial guess $x^{(0)}$ if and only if $\alpha^{2}+x^{*}(\mu \mathcal{S}+\mathscr{H}) x>0$, where $\mu=x^{*} S x / x^{*} H x$, $H=\left(A^{*}+A\right) / 2, S=\left(A-A^{*}\right) / 2 i, \mathscr{H}=\left[(\mathscr{L} \mathcal{U})^{*}+\mathscr{L} \mathcal{U}\right] / 2, \mathcal{S}=$ $\left[\mathscr{L} \mathcal{U}-(\mathscr{L} \mathcal{U})^{*}\right] / 2 i$, and $x \in\left\{x \in \mathbb{C}^{n}: T_{\alpha} x=\lambda x, x^{*} x=\right.$ 1 and $\left.|\lambda|=\rho\left(T_{\alpha}\right)\right\}$.

Proof. Since the iterative scheme (3) is convergent if and only if the iterative scheme (4) is convergent, it follows from [17] that (4) converges for any given $x^{(0)}$ if and only if $\rho\left(T_{\alpha}\right)<$ 1 , where $\rho\left(T_{\alpha}\right)$ denotes the spectral radius of the matrix $T_{\alpha}$. Assume $x \in\left\{x \in \mathbb{C}^{n}: T_{\alpha} x=\lambda x, x^{*} x=1\right.$ and $\left.|\lambda|=\rho\left(T_{\alpha}\right)\right\}$. Then $T_{\alpha} x=\lambda x$. It follows from Lemma 1 that

$$
\left(\alpha^{2} I-\alpha A+\mathscr{L} \mathcal{U}\right) x=\lambda\left(\alpha^{2} I+\alpha A+\mathscr{L} \mathcal{U}\right) x .
$$

Multiplying (7) on the left side by $x^{*}$,

$$
x^{*}\left(\alpha^{2} I-\alpha A+\mathscr{L} \mathscr{U}\right) x=\lambda x^{*}\left(\alpha^{2} I+\alpha A+\mathscr{L} \mathcal{U}\right) x .
$$

We assert $\phi:=x^{*}\left(\alpha^{2} I+\alpha A+\mathscr{L} \mathcal{U}\right) x \neq 0$. Otherwise, $\phi=$ $x^{*}\left(\alpha^{2} I+\alpha A+\mathscr{L} \mathcal{U}\right) x=0$, and consequently $\varphi:=x^{*}\left(\alpha^{2} I-\right.$ $\alpha A+\mathscr{L} \mathscr{U}) x=0$. As a result,

$$
\begin{aligned}
& \operatorname{Re}(\phi)=x^{*}\left(\alpha^{2} I+\alpha H+\mathscr{H}\right) x=0, \\
& \operatorname{Re}(\varphi)=x^{*}\left(\alpha^{2} I-\alpha H+\mathscr{H}\right) x=0 .
\end{aligned}
$$

Equations (9) yield that

$$
\operatorname{Re}(\phi)-\operatorname{Re}(\varphi)=2 \alpha x^{*} H x=0 .
$$

Equation (10) shows that $H=\left(A^{*}+A\right) / 2$ is not Hermitian positive definite; that is, $A$ is not non-Hermitian positive definite. Therefore, a contradiction appears to indicate $\phi=$ $x^{*}\left(\alpha^{2} I+\alpha A+\mathscr{L} \mathcal{U}\right) x \neq 0$. Thus,

$$
\begin{aligned}
\rho\left(T_{\alpha}\right) & =|\lambda|=\frac{\left|x^{*}\left(\alpha^{2} I-\alpha A+\mathscr{L} \mathcal{U}\right) x\right|}{\left|x^{*}\left(\alpha^{2} I+\alpha A+\mathscr{L} \mathcal{U}\right) x\right|} \\
& =\frac{\sqrt{\operatorname{Re}^{2}(\varphi)+\operatorname{Im}^{2}(\varphi)}}{\sqrt{\operatorname{Re}^{2}(\phi)+\operatorname{Im}^{2}(\phi)}} \\
& =\sqrt{\frac{\operatorname{Re}^{2}(\varphi)+\operatorname{Im}^{2}(\varphi)}{\operatorname{Re}^{2}(\phi)+\operatorname{Im}^{2}(\phi)}},
\end{aligned}
$$

which indicates that $\rho\left(T_{\alpha}\right)<1$ if and only if

$$
\begin{aligned}
& {\left[\operatorname{Re}^{2}(\phi)+\operatorname{Im}^{2}(\phi)\right]-\left[\operatorname{Re}^{2}(\varphi)+\operatorname{Im}^{2}(\varphi)\right]} \\
& \quad=4 \alpha x^{*} H x\left[\alpha^{2}+x^{*}(\mathscr{H}+\mu \mathcal{S}) x\right] \\
& \quad>0,
\end{aligned}
$$

where $\mu=x^{*} S x / x^{*} H x, H=\left(A^{*}+A\right) / 2, S=\left(A-A^{*}\right) / 2 i$, $\mathscr{H}=\left[(\mathscr{L} \mathcal{U})^{*}+\mathscr{L} \mathscr{U}\right] / 2$, and $\mathcal{S}=\left[\mathscr{L} \mathcal{U}-(\mathscr{L} \mathcal{U})^{*}\right] / 2 i$. Since $\alpha>0$ and $x^{*} H x>0$, (12) holds if and only if $\alpha^{2}+x^{*}(\mathscr{H}+$ $\mu \delta) x>0$. This completes the proof. 
Theorem 2 presents a convergence condition for the TTS method. But, in fact, this condition is difficult to be applied. It is necessary to give a practical condition. It follows from Theorem 2 that we can get the following conclusion.

Theorem 3. Let $A \in \mathbb{C}^{n \times n}$ be non-Hermitian positive definite with the TTS as in (2), and let

$$
\tau=\min \left\{\lambda_{\min }\left(H^{-1} S\right) \lambda_{\max }(\delta), \lambda_{\max }\left(H^{-1} S\right) \lambda_{\min }(\delta)\right\},
$$

where $\lambda_{\min }(C)$ and $\lambda_{\max }(C)$ denote the minimal and maximal eigenvalues of the matrix $C$, respectively, $H=\left(A^{*}+A\right) / 2, S=$ $\left(A-A^{*}\right) / 2 i, \mathscr{H}=\left[(\mathscr{L} \mathscr{U})^{*}+\mathscr{L} \mathscr{U}\right] / 2$, and $\delta=[\mathscr{L} \mathscr{U}-$ $\left.(\mathscr{L} \mathscr{U})^{*}\right] / 2 i$. If $\alpha^{2}+\lambda_{\text {min }}(\mathscr{H})+\tau>0$, then the TTS method converges to the unique solution of (1) for any choice of the initial guess $x^{(0)}$.

Although the TTS method does not always converge for non-Hermitian positive definite linear systems, it always converges for Hermitian positive definite linear systems.

Theorem 4. Let $A \in \mathbb{C}^{n \times n}$ be Hermitian positive definite with the TTS as in (2). Then, for all $\alpha>0$, the TTS method converges to the unique solution of (1) for any choice of the initial guess $x^{(0)}$. Furthermore, it holds that

$$
\begin{gathered}
\alpha_{o p t}=\arg \min _{\alpha>0}\left\{\rho\left(T_{\alpha}\right)\right\}=\sqrt{x^{*} \mathscr{L} \mathscr{L}^{*} x} \in\left[\sigma_{n}, \sigma_{1}\right], \\
\rho\left(T_{\alpha_{o p t}}\right)=\frac{2 \sqrt{x^{*} \mathscr{L} \mathscr{L}^{*} x}-x^{*} A x}{2 \sqrt{x^{*} \mathscr{L} \mathscr{L}^{*} x}+x^{*} A x},
\end{gathered}
$$

where $x \in\left\{x \in \mathbb{C}^{n}: T_{\alpha} x=\lambda x, x^{*} x=1\right.$ and $\left.|\lambda|=\rho\left(T_{\alpha}\right)\right\}$ and $\sigma_{n}$ and $\sigma_{1}$ are the minimal and maximal singular values of $\mathscr{L}$, respectively.

Proof. Since $A$ is Hermitian positive definite, $A=\mathscr{L}+\mathscr{U}=$ $\mathscr{L}+\mathscr{L}^{*}$, and $\mathscr{L} \mathscr{U}=\mathscr{L}^{*}$ is Hermitian positive definite. Following (11),

$$
\begin{aligned}
\rho\left(T_{\alpha}\right) & =\lambda=\frac{x^{*}\left(\alpha^{2} I-\alpha A+\mathscr{L} \mathscr{U}\right) x}{x^{*}\left(\alpha^{2} I+\alpha A+\mathscr{L} \mathscr{U}\right) x} \\
& =\frac{\alpha^{2}-\alpha x^{*} A x+x^{*} \mathscr{L} \mathscr{L}^{*} x}{\alpha^{2}+\alpha x^{*} A x+x^{*} \mathscr{L} \mathscr{L}^{*} x} \\
& <1,
\end{aligned}
$$

which shows the convergence of the TTS method. Following (15)

$$
\begin{aligned}
\rho\left(T_{\alpha}\right) & =\frac{\alpha^{2}-\alpha x^{*} A x+x^{*} \mathscr{L} \mathscr{L}^{*} x}{\alpha^{2}+\alpha x^{*} A x+x^{*} \mathscr{L} \mathscr{L}^{*} x} \\
& =1-\frac{2 \alpha x^{*} A x}{\alpha^{2}+\alpha x^{*} A x+x^{*} \mathscr{L} \mathscr{L}^{*} x} \\
& =1-f(\alpha),
\end{aligned}
$$

where $f(\alpha)=2 \alpha x^{*} A x /\left(\alpha^{2}+\alpha x^{*} A x+x^{*} \mathscr{L}^{*} x\right)$. As a result,

$$
\min _{\alpha>0}\left\{\rho\left(T_{\alpha}\right)\right\}=1-\max _{\alpha>0} f(\alpha) .
$$

Since

$$
f^{\prime}(\alpha)=\frac{2 x^{*} A x\left(x^{*} \mathscr{L}^{*} x-\alpha^{2}\right)}{\left(\alpha^{2}+\alpha x^{*} A x+x^{*} \mathscr{L} \mathscr{L}^{*} x\right)^{2}},
$$

$f(\alpha)$ is gradually increasing if $\alpha \in\left(0, \sqrt{x^{*} \mathscr{L} \mathscr{L}^{*} x}\right), f(\alpha)$ is gradually decreasing if $\alpha \in\left(\sqrt{x^{*} \mathscr{L} \mathscr{L}^{*} x}, \infty\right)$, and, consequently, when $\alpha=\sqrt{x^{*} \mathscr{L} \mathscr{L}^{*} x}, f(\alpha)$ gets its maximum $\max _{\alpha>0} f(\alpha)=2 \alpha x^{*} A x /\left(x^{*} A x+2 \sqrt{x^{*} \mathscr{L}^{*} x}\right)$. Therefore, when

$$
\begin{gathered}
\alpha_{\text {opt }}=\arg \min _{\alpha>0}\left\{\rho\left(T_{\alpha}\right)\right\}=\sqrt{x^{*} \mathscr{L} \mathscr{L}^{*} x} \in\left[\sigma_{n}, \sigma_{1}\right], \\
\rho\left(T_{\alpha_{\text {opt }}}\right)=\frac{2 \sqrt{x^{*} \mathscr{L} \mathscr{L}^{*} x}-x^{*} A x}{2 \sqrt{x^{*} \mathscr{L} \mathscr{L}^{*} x}+x^{*} A x},
\end{gathered}
$$

which shows that we completed the proof.

\section{Remark on and Comparison of Convergence Theorems}

In fact, the TTS method is a special case of the generalized assymmetric SOR iteration method with parameter matrices when specifically choosing the parameter matrices. This scheme is called the ALUS method in $[18,19]$.

The ALUS Method. Given an initial guess $x^{(0)}$, for $k=$ $0,1,2, \ldots$, until $\left\{x^{(k)}\right\}$ converges, compute

$$
\begin{gathered}
(\alpha I+\widetilde{\mathscr{L}}) x^{(k+1 / 2)}=(\alpha I-\widetilde{\mathscr{U}}) x^{(k)}+b, \\
(\alpha I+\widetilde{\mathscr{U}}) x^{(k+1)}=(\alpha I-\widetilde{\mathscr{L}}) x^{(k+1 / 2)}+b,
\end{gathered}
$$

where $\alpha$ is a given positive constant, $\widetilde{\mathscr{L}}=D_{1}+L, \widetilde{\mathscr{U}}=D_{2}+U$, $D_{1}+D_{2}=D$, and $L, U$, and $D$ are defined in (3).

We easily generalize the convergence theorems on TTS method to ALUS method.

Theorem 5. Let $A \in \mathbb{C}^{n \times n}$ be non-Hermitian positive definite with the ALUS as in (20). Then the ALUS method converges to the unique solution of (1) for any choice of the initial guess $x^{(0)}$ if and only if $\alpha^{2}+x^{*}(\mu \mathcal{S}+\mathscr{H}) x>0$, where $\mu=x^{*} S x / x^{*} H x$, $H=\left(A^{*}+A\right) / 2, S=\left(A-A^{*}\right) / 2 i, \mathscr{H}=\left[(\widetilde{\mathscr{L}} \widetilde{\mathcal{U}})^{*}+\widetilde{\mathscr{L}} \widetilde{\mathcal{U}}\right] / 2, \mathcal{S}=$ $\left[\widetilde{\mathscr{L}} \widetilde{U}-(\widetilde{\mathscr{L}} \widetilde{U})^{*}\right] / 2 i$, and $x \in\left\{x \in \mathbb{C}^{n}: T_{\alpha} x=\lambda x, x^{*} x=\right.$ 1 and $\left.|\lambda|=\rho\left(T_{\alpha}\right)\right\}$.

Theorem 6. Let $A \in \mathbb{C}^{n \times n}$ be non-Hermitian positive definite with the ALUS as in (20), and let

$$
\tau=\min \left\{\lambda_{\min }\left(H^{-1} S\right) \lambda_{\max }(\mathcal{S}), \lambda_{\max }\left(H^{-1} S\right) \lambda_{\min }(\mathcal{S})\right\},
$$

where $\lambda_{\min }(C)$ and $\lambda_{\max }(C)$ denote the minimal and maximal eigenvalues of the matrix $C$, respectively, $H=\left(A^{*}+A\right) / 2, S=$ $\left(A-A^{*}\right) / 2 i, \mathscr{H}=\left[(\widetilde{\mathscr{L}} \widetilde{\mathscr{U}})^{*}+\widetilde{\mathscr{L}} \widetilde{\mathcal{U}}\right] / 2$, and $\mathcal{S}=[\widetilde{\mathscr{L}} \widetilde{\mathscr{U}}-$ $\left.(\widetilde{\mathscr{U}} \widetilde{U})^{*}\right] / 2 i$. If $\alpha^{2}+\lambda_{\text {min }}(\mathscr{H})+\tau>0$, then the ALUS method converges to the unique solution of (1) for any choice of the initial guess $x^{(0)}$. 
The proofs of Theorems 5 and 6 directly result from the proofs of Theorems 2 and 3.

Like Theorem 4 in [18] or Theorem 6 in [19], Theorem 5 has only theoretical significance since it is difficult to be applied. However, Theorem 6, along with Theorem 3 in [18] or Theorem 4 in [19], proposes a practical condition on convergence of ALUS method. But the condition in Theorem 6 is wider than Theorem 3 in [18] or Theorem 4 in [19]. The following will give an example to demonstrate this fact.

Example 7. The coefficient matrix $A$ of linear system (1) is given as

$$
A=\left[\begin{array}{cc}
1 & 2 \\
-2 & 1
\end{array}\right]
$$

Now, we consider solving this system by ALUS method. Let $D_{1}=\left[\begin{array}{ll}x & 0 \\ 0 & y\end{array}\right]$. Then $D_{2}=D-D_{1}=\left[\begin{array}{cc}1-x & 0 \\ 0 & 1-y\end{array}\right], \widetilde{\mathscr{L}}=D_{1}+L=$ $\left[\begin{array}{cc}x & 0 \\ -2 & y\end{array}\right]$, and $\widetilde{\mathscr{U}}=D_{2}+U=\left[\begin{array}{cc}1-x & 2 \\ 0 & 1-y\end{array}\right]$. In order that the ALUS method converges to the unique solution of (1) for any choice of the initial guess $x^{(0)}$ Theorem 3 in [18] or Theorem 4 in [19] shows that $\widetilde{\mathscr{L}}+\widetilde{\mathscr{L}}^{*}=\left[\begin{array}{cc}2 x & -2 \\ -2 & 2 y\end{array}\right]$ and $\widetilde{\mathscr{U}}+\widetilde{\mathscr{U}}^{*}=\left[\begin{array}{cc}2(2-x) & 2 \\ 2 & 2(1-y)\end{array}\right]$ are both positive definite for all $x \in(0,1)$ and $y \in(0,1)$. As a result, the set

$$
(x, y) \in \mathbb{R} \times \mathbb{R}:\left\{\begin{array}{l}
x y>1, \\
x y>x+y, \\
x \in(0,1), y \in(0,1)
\end{array}\right.
$$

is not empty. In fact, the set (23) is empty since the inequality $x y>1$ fails to hold when $x \in(0,1)$ and $y \in(0,1)$. Therefore, Theorem 3 in [18] or Theorem 4 in [19] does not give the convergence of the ALUS method if we solve the linear system (1) with the coefficient matrix $A$ in (22).

Using Theorem 6 , set $x=y=1 / 2$ and compute $\tau=-2$ and $\lambda_{\min }(\mathscr{H})=-15 / 4$. Then when $\alpha>\sqrt{23} / 2$ the ALUS method converges to the unique solution of (1) for any choice of the initial guess $x^{(0)}$.

Example 8. Linear system (1) is shifted skew-Hermitian linear system (see [7-14]) whose coefficient matrix $A$ is given as

$$
A=\gamma I+S
$$

where $\gamma$ is a positive constant, $I$ is the $n \times n$ identity matrix, and

$$
S=L-L^{*}
$$

is a skew-Hermitian matrix with $L$ a lower triangular matrix.

The shifted skew-Hermitian linear system, arising in the HSS iterative method, can be much more problematic; in some cases this solution is as difficult as that of the original linear system [10]. Since HSS method fails to solve this linear system, we consider the ALUS method and TTS method.

We assert that if $\gamma \leq \lambda_{\max }(G)-\lambda_{\min }(G)$ with $G=L+L^{*}$ and $\lambda_{\max }(G)$ and $\lambda_{\min }(G)$ being the maximum and minimum eigenvalues of the matrix $G$, respectively, Theorem 3 in [18] or Theorem 4 in [19] fails to give the convergence of the ALUS method when solving the skew-Hermitian linear system (1).

Let $D_{1}=x I$. Then $D_{2}=D-D_{1}=(\gamma-x) I, \widetilde{\mathscr{L}}=x I+L$, and $\widetilde{\mathscr{U}}=(\gamma-x) I-L^{*}$. In order that the ALUS method converges to the unique solution of (1) for any choice of the initial guess $x^{(0)}$ Theorem 3 in [18] or Theorem 4 in [19] shows that $\widetilde{\mathscr{L}}+$ $\widetilde{\mathscr{L}}^{*}=2 x I+G$ and $\widetilde{\mathscr{U}}+\widetilde{\mathscr{U}}^{*}=2(\gamma-x) I-G$ are both positive definite for all $x \in(0, \gamma)$. Thus, it is easy to get

$$
2 x+\lambda_{\min }(G)>0, \quad 2(\gamma-x)-\lambda_{\max }(G)>0 .
$$

It follows from (26) that $\gamma>\lambda_{\max }(G)-\lambda_{\text {min }}(G)$ which shows that when $\gamma \leq \lambda_{\max }(G)-\lambda_{\min }(G)$ Theorem 3 in [18] or Theorem 4 in [19] fails to give the convergence of the ALUS method.

We consider using Theorem 6. Compute $\tau=$ $\lambda_{\max }(-i S) \lambda_{\min }(-i S) / 2$. Theorem 6 shows that if $f(x)=\alpha^{2}+$ $x(\gamma-x)-\lambda_{\max }\left(L L^{*}\right)+(\gamma / 2-x) \lambda_{\min }(G)>0$ the ALUS method converges to the unique solution of (1) for any choice of the initial guess $x^{(0)}$. Furthermore,

$$
\max _{x \in(0, \gamma)} f(x)=\alpha^{2}+\frac{\left(\gamma^{2}+\lambda_{\min }^{2}(G)\right)}{4}-\lambda_{\max }\left(L L^{*}\right)
$$

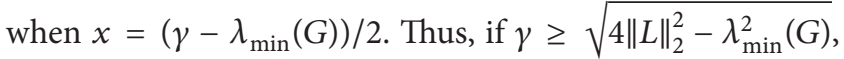
Theorem 6 shows that the ALUS method converges to the unique solution of (1) for any choice of the initial guess $x^{(0)}$ and for all $\alpha>0$; otherwise, it follows from Theorem 6 that the ALUS method converges to the unique solution of (1) for any choice of the initial guess $x^{(0)}$ and for all $\alpha>$ $\sqrt{\|L\|_{2}^{2}-\left(\gamma^{2}+\lambda_{\min }^{2}(G)\right) / 4}$.

In particular, if $\gamma \geq 2\|L\|_{2}$, Theorem 3 yields that the TTS method converges to the unique solution of (1) for any choice of the initial guess $x^{(0)}$ and for all $\alpha>0$; otherwise, it follows from Theorem 3 that the TTS method converges to the unique solution of (1) for any choice of the initial guess $x^{(0)}$ and for all $\alpha>\sqrt{\|L\|_{2}^{2}-\gamma^{2} / 4}$.

\section{Numerical Examples}

In this section we describe the results of a numerical simple example with the TTS method on a set of linear systems arising from a finite element discretization of a convectiondiffusion equation in two dimensions.

Example 1. The coefficient matrix $A$ of linear system (1) is given as

$$
A=\left[\begin{array}{cccc}
1 & 1.1 & 1.2 & 1 \\
-0.5 & 1 & 1.4 & 0.65 \\
-0.2 & -0.6 & 1 & 1.55 \\
0.2 & 0.25 & -0.45 & 1
\end{array}\right]
$$

Now, we investigate convergence of TTS method for linear system (1) by Theorem 3 . It is known that $A$ is nonHermitian positive definite. By Matlab computations, we 
TABLE 1: The comparison results of $\rho\left(T_{\alpha}\right)$ with different parameter pairs $\alpha$.

\begin{tabular}{|c|c|c|c|c|c|c|c|c|}
\hline $\bar{\alpha}$ & $10^{-5}$ & $10^{-4}$ & $10^{-3}$ & $10^{-2}$ & 0.1 & 0.5 & 1 & 1.1 \\
\hline$\rho\left(T_{\alpha}\right)$ & 1.0000 & 0.9999 & 0.9994 & 0.9940 & 0.9435 & 0.8476 & 0.7163 & 0.6622 \\
\hline$\alpha$ & 1.2 & 1.3 & 1.4 & 1.5 & 1.6 & 1.7 & 1.8 & 1.9 \\
\hline$\rho\left(T_{\alpha}\right)$ & 0.7795 & 0.8983 & 0.9422 & 0.9455 & 0.9199 & 0.8698 & 0.7921 & 0.6381 \\
\hline$\alpha$ & 2 & 2.25 & 2.5 & 3 & 3.05 & 3.1 & 3.15 & 3.2 \\
\hline$\rho\left(T_{\alpha}\right)$ & 0.6193 & 0.5784 & 0.5498 & 0.5283 & 0.5281 & 0.5282 & 0.5285 & 0.5290 \\
\hline$\alpha$ & 3.3 & 3.4 & 3.5 & 4 & 4.5 & 5 & 10 & 15 \\
\hline$\rho\left(T_{\alpha}\right)$ & 0.5307 & 0.5330 & 0.5358 & 0.5550 & 0.5779 & 0.6012 & 0.7580 & 0.8292 \\
\hline$\alpha$ & 20 & 30 & 50 & 100 & 150 & $10^{3}$ & $10^{4}$ & $10^{5}$ \\
\hline$\rho\left(T_{\alpha}\right)$ & 0.8694 & 0.9099 & 0.9449 & 0.9720 & 0.9813 & 0.9972 & 0.9997 & 1.0000 \\
\hline
\end{tabular}

get $\lambda_{\min }(\mathscr{H})+\tau=-3.0471$. As a result, when $\alpha \epsilon$ $(1.7456,+\infty)$, the TTS method theoretically converges to the unique solution of (1) for any choice of the initial guess $x^{(0)}$. But, when $\alpha \rightarrow+\infty$, this method either converges very slowly or fails to converge since $\rho\left(T_{\alpha}\right) \rightarrow 1$.

By numerical experiments on Matlab program, one has Table 1 .

Table 1 shows that, for given matrix $A$, the TTS method converges for $\alpha \in\left(10^{-5}, 10^{5}\right)$. Further, $\rho\left(T_{\alpha}\right)$ is gradually decreasing when $\alpha \in(0,1.1)$ and $\alpha \in(1.5,3.05)$ while it is gradually increasing when $\alpha \in(1.1,1.5)$ and $\alpha \in(3.05,+\infty)$. Thus, $\rho\left(T_{\alpha}\right)$ has two minimal values: $\rho\left(T_{1.1}\right)=0.6622$ and $\rho\left(T_{3.05}\right)=0.5281$. However, $\rho\left(T_{3.05}\right)=0.5281$ is the minimal value. As a result, $\alpha_{\text {opt }}=3.05$. It follows from Table 1 that Theorem 3 is true. Furthermore, the interval obtained in Theorem 3 includes the optimal point $\alpha_{\text {opt }}=3.05$.

\section{Conclusions}

This paper studies convergence of TTS and ALUS iterative methods for non-Hermitian positive definite linear systems. Some sufficient and necessary conditions for convergence are proposed. But these conditions are only theoretically significant and difficult to apply to practical computations. In what follows, several conditions are presented such that the TTS method and ALUS method converge for non-Hermitian positive definite linear systems.

\section{Conflict of Interests}

The authors declare that there is no conflict of interests regarding the publication of this paper.

\section{Acknowledgments}

Cheng-Yi Zhang was partly supported by the Science Foundation of the Education Department of Shaanxi Province of China (2013JK0593), the Scientific Research Foundation (BS1014) and the Education Reform Foundation (2012JG40) of Xi'an Polytechnic University, and the National Natural Science Foundations of China (11201362 and 11271297); YuQian Yang was partly supported by the National Natural Science Foundation of China (no. 61201297); Qiang Sun was partly supported by the National Natural Science Foundation of China (no. 61001140) and the Scientific Research Program
Funded by Shaanxi Provincial Education Department (no. 12JK0544).

\section{References}

[1] H. Elman, D. Silvester, and A. Wathen, Finite Elements and Fast Iterative Solvers with Applications in Incompressible Fluid Dynamics, Numerical Mathematics and Scientific Computation, Oxford University Press, Oxford, UK, 2005.

[2] M. Benzi and M. K. Ng, "Preconditioned iterative methods for weighted toeplitz least squares problems," SIAM Journal on Matrix Analysis and Applications, vol. 27, no. 4, pp. 1106-1124, 2006.

[3] M. Benzi and D. Bertaccini, "Block preconditioning of realvalued iterative algorithms for complex linear systems," IMA Journal of Numerical Analysis, vol. 28, no. 3, pp. 598-618, 2008.

[4] A. Berman and R. J. Plemmons, Nonnegative Matrices in the Mathematical Sciences, Academic Press, New York, NY, USA, 1979, Reprinted by SIAM, Philadelphia, Pa, USA, 1994.

[5] C.-L. Wang and Z.-Z. Bai, "Sufficient conditions for the convergent splittings of non-Hermitian positive definite matrices," Linear Algebra and Its Applications, vol. 330, no. 1-3, pp. 215-218, 2001.

[6] L. Wang and Z.-Z. Bai, "Convergence conditions for splitting iteration methods for non-Hermitian linear systems," Linear Algebra and Its Applications, vol. 428, no. 2-3, pp. 453-468, 2008.

[7] Z.-Z. Bai, G. H. Golub, and M. K. Ng, "Hermitian and Skew-Hermitian splitting methods for non-Hermitian positive definite linear systems," SIAM Journal on Matrix Analysis and Applications, vol. 24, no. 3, pp. 603-626, 2003.

[8] Z.-Z. Bai, G. H. Golub, and M. K. Ng, "On successive-overrelaxation acceleration of the Hermitian and Skew-Hermitian splitting iterations," Numerical Linear Algebra with Applications, vol. 14, no. 4, pp. 319-335, 2007.

[9] Z.-Z. Bai, G. H. Golub, and J.-Y. Pan, "Preconditioned Hermitian and Skew-Hermitian splitting methods for non-Hermitian positive semidefinite linear systems," Numerische Mathematik, vol. 98, no. 1, pp. 1-32, 2004.

[10] M. Benzi, "A Generalization of the he rmitian and Skewhermitian splitting iteration," SIAM Journal on Matrix Analysis and Applications, vol. 31, no. 2, pp. 360-374, 2009.

[11] Z.-Z. Bai, G. H. Golub, L.-Z. Lu, and J.-F. Yin, "Block triangular and Skew-Hermitian splitting methods for positive-definite linear systems," SIAM Journal on Scientific Computing, vol. 26, no. 3, pp. 844-863, 2005.

[12] Z.-Z. Bai, G. H. Golub, and M. K. Ng, "On inexact hermitian and Skew-Hermitian splitting methods for non-Hermitian positive 
definite linear systems," Linear Algebra and Its Applications, vol. 428, no. 2-3, pp. 413-440, 2008.

[13] L. Li, T. -Z. Huang, and X. -P. Liu, "Modified Hermitian and Skew-Hermitian splitting methods for non-Hermitian positivedefinite linear systems," Numerical Linear Algebra With Applications, vol. 14, no. 3, pp. 217-235, 2007.

[14] Z.-Z. Bai and G. H. Golub, "Accelerated Hermitian and SkewHermitian splitting iteration methods for saddle-point problems," IMA Journal of Numerical Analysis, vol. 27, no. 1, pp. 1-23, 2007.

[15] M. Benzi, M. J. Gander, and G. H. Golub, "Optimization of the Hermitian and Skew-Hermitian splitting iteration for saddlepoint problems," BIT Numerical Mathematics, vol. 43, no. 5, pp. 881-900, 2003.

[16] M. Benzi and G. H. Golub, "A preconditioner for generalized saddle point problems," SIAM Journal on Matrix Analysis and Applications, vol. 26, no. 1, pp. 20-41, 2005.

[17] R. S. Varga, Matrix Iterative Analysis, Springer, Berlin, Germany, 2nd edition, 2000.

[18] Q. Xiang and S.-P. Wu, "A modified alternating direction method for positive definite systems," in Information Engneering and Applications, R.-B. Zhu and Y. Ma, Eds., vol. 154 of Lecture Notes in Electrical Engineering, pp. 437-444, 2012.

[19] Q. Xiang, S. -P. Wu, and Y. Xu, "Alternating lower-upper splitting iterative method and its application," Journal of Beijing University of Aeronautics and Astronautics, vol. 38, pp. 953-956, 2012 (Chinese). 


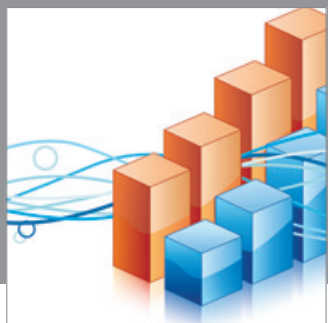

Advances in

Operations Research

mansans

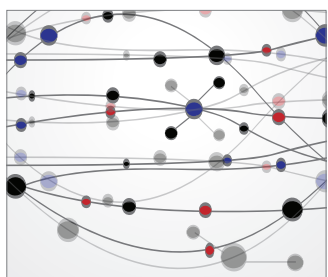

The Scientific World Journal
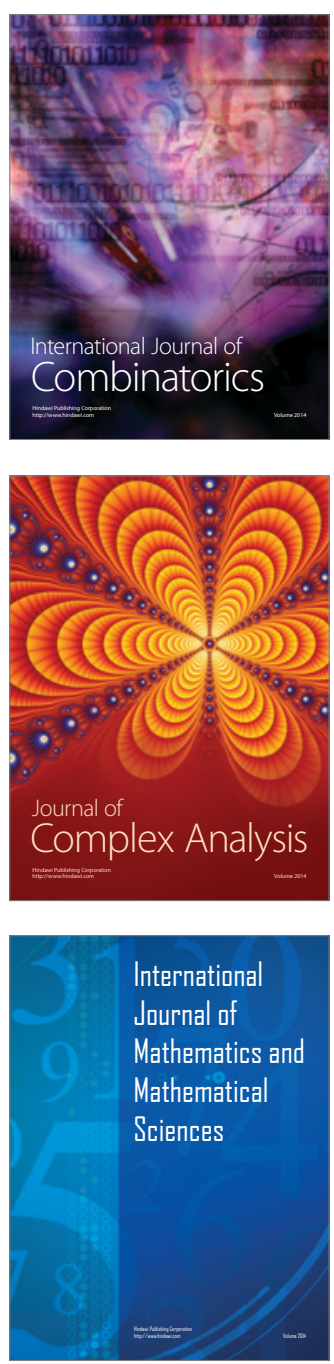
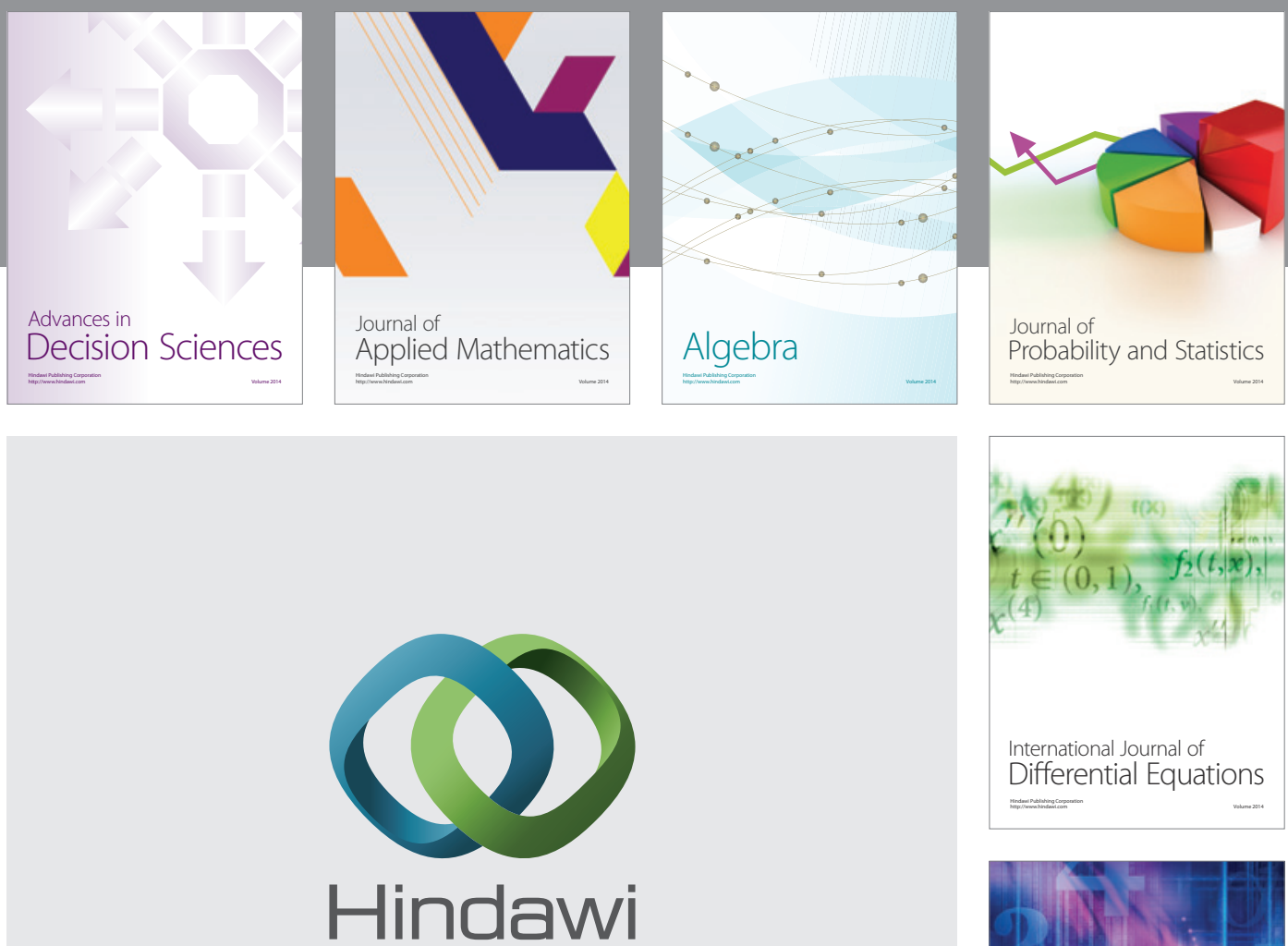

Submit your manuscripts at http://www.hindawi.com
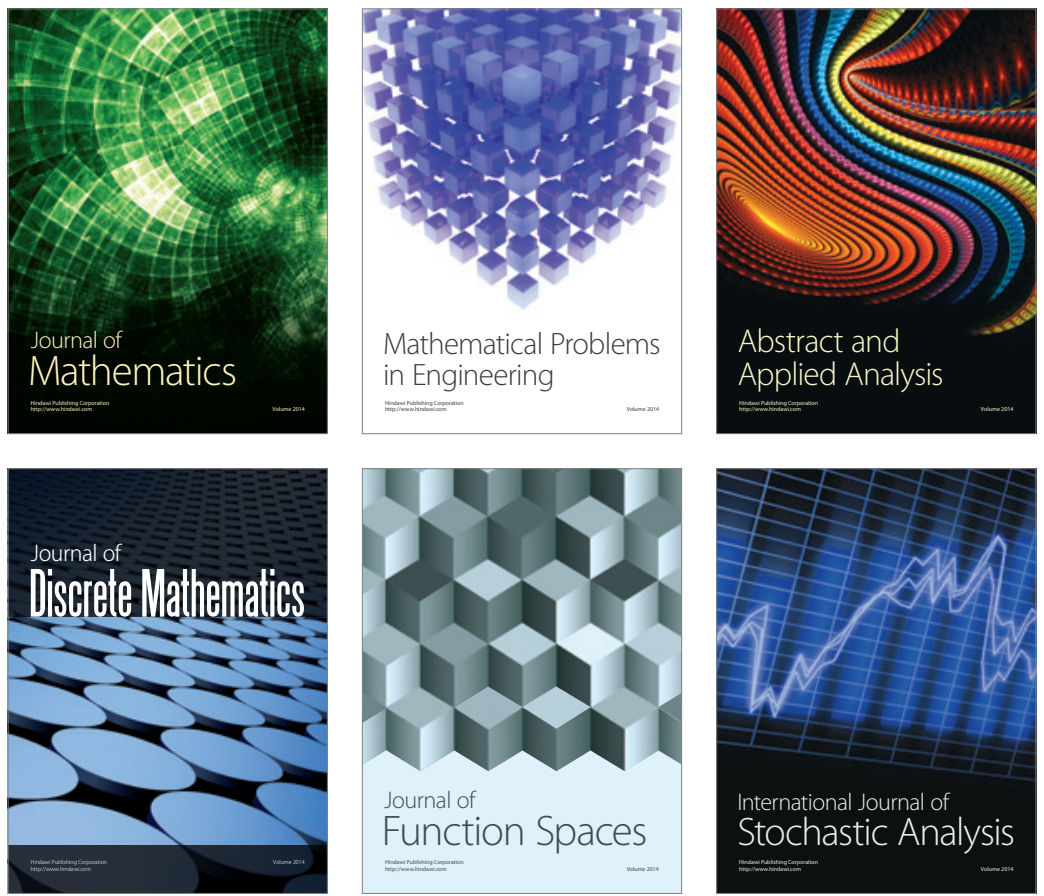

Journal of

Function Spaces

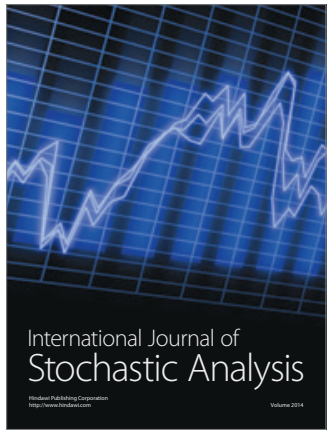

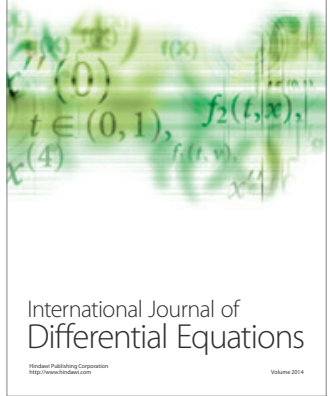
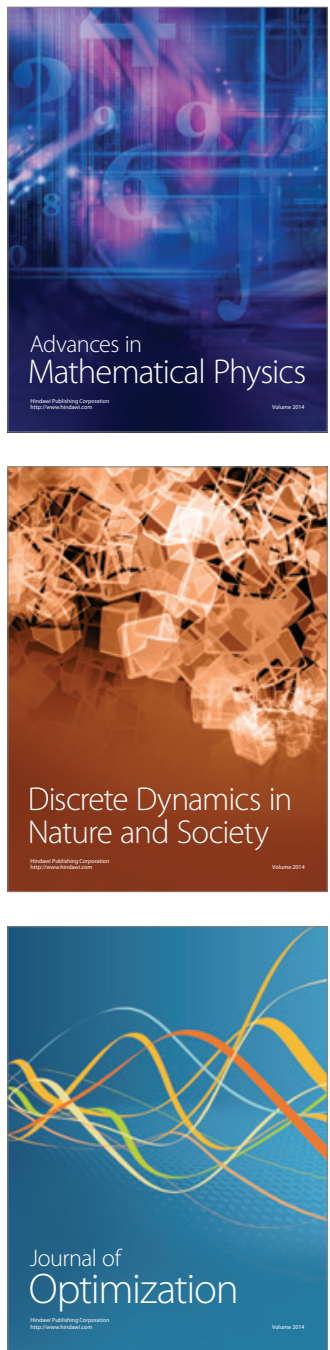